\title{
OPINION
}

\section{Personalized Medicine: A Perk of Privilege?}

\author{
JJ Griggs 1
}

\begin{abstract}
Advances in molecular testing and genomic technology offer promise in helping identify people at risk of developing disease and those most (or least) likely to benefit from risk reduction and treatment strategies. Yet not all of those who are eligible for such technologies have access to the benefits of these advances. Given the inequities in our health-care system, there is no assurance that expanding research into molecular and genomic testing will benefit everyone equally. Moreover, widespread adoption of new and emerging technologies poses challenges for an already overtaxed health-care system.
\end{abstract}

Consider a 48-year-old premenopausal law professor who is diagnosed with a $2.2-\mathrm{cm}$ breast cancer that is positive for hormone receptor and negative for human epidermal growth factor receptor-2, with grade 2 histology. Because of a family history of early-onset breast cancer in a paternal aunt and her sister, she is advised to have genetic counseling and then testing for an inherited susceptibility to breast and ovarian cancer. She is found not to have a mutation in either BRCA1 or BRCA2. She undergoes a sentinel lymph node procedure that confirms the absence of lymph node involvement. Her oncologist recommends that, after approval has been secured from her insurance company, her primary tumor be sent for a 21-gene assay. This identifies the cancer as having a low risk score, indicating that chemotherapy will not improve the likelihood of disease-free and overall survival. She may have blood sent for CYP2D6 genotyping if her oncologist, who attends the annual meeting of the national oncology society, believes this will help predict the benefit of tamoxifen. She will most likely be treated with breast-conserving therapy, adjuvant radiation therapy, and tamoxifen. This patient has certainly benefited from what is commonly called personalized medicine, an emerging science that allows tailoring of treatment according to the characteristics of host and disease.

Imagine now that, instead of having insurance through her employer, she has been laid off from a part-time job at a large retail store and has recently joined the ranks of the nearly 46 million uninsured Americans, or that she has Medicaid. She cannot pay for genetic counseling and testing. Concerned that she may have inherited a susceptibility to breast cancer, she elects to have bilateral mastectomy. Medicaid does not pay for the 21-gene assay in all but a handful of states, so she receives adjuvant chemotherapy followed by tamoxifen, CYP2D6 genotyping not even being considered by her oncologist. Based on her insurance status alone, she has not shared the benefits of advances in personalized medicine in the management of her disease.

The literature is replete with studies demonstrating health disparities among people of lower socioeconomic status (SES) - whether SES is measured according to individual-level income and education or by neighborhood socioeconomic variables. Although health disparities are due only in part to inequities in medical care, addressing the gaps in quality of care is obviously a worthwhile endeavor. It follows that correcting disparities in access to molecular and genomic technologies-an increasingly important aspect of medical care-will remedy, in part, disparities in the quality of care. Conversely, failing to do so will inevitably widen the differences between the haves and have-nots. The principle of distributive justice requires that the benefits of medical advances be shared equally by all people. ${ }^{1}$ As illustrated in the aforementioned case, access to molecular and genomic technologies varies according to insurance status, which in turn varies according to SES.

Lack of payment for molecular and genomic tests is only one barrier to full and equal access. Provider awareness and patient decision support are two other factors in determining who benefits from application of new specialized tests. People with fewer economic resources are less likely to receive preventive services of all types, including risk reduction and screening services, and are less likely to have a usual source of medical care. Economic insecurity places competing demands on patients and their families, often leaving health care low on the list of priorities. Lower SES may also be associated with receipt of care from providers whose practices

1Division of Hematology/Oncology, Department of Medicine, University of Michigan, Ann Arbor, Michigan, USA. Correspondence: JJ Griggs (jengrigg@umich.edu) 
have fewer resources, such as access to certified genetic counselors or other forms of education and patient decision support. Clearly, improving provider knowledge and understanding of genomic medicine is a critical component of any effort designed to effect change in care. Implementing educational programs for providers requires novel approaches for reaching practicing providers and those in training.

In addition, decision making about genetic testing and other molecular and genomic tests is far from simple. Understanding the concepts of probabilities, relative and absolute risk reduction, and short- and long-term consequences of testing or not testing does not come easily, even to medical providers. Health literacy and numeracy levels, low enough to present a barrier to informed decision making even among those with high levels of education, pose even greater barriers in people with lower levels of educational attainment. ${ }^{2}$ The stress associated with cancer and other serious illnesses is likely to further impede understanding and decision making. Decision aids, which can help elicit and clarify people's preferences, may be particularly useful among the medically underserved. Development and dissemination of informational support, including decision aids, should thus be carried out in parallel with development of novel molecular markers in order to help patients and their providers make the most of advances in molecular and genomic medicine.

Expanding access to molecular and genomic technologies to people of limited economic resources, including people in less wealthy countries, will allow more people to benefit from scientific advances and may reduce the likelihood of overtreatment, the risk of adverse drug reactions, and the costs of care for an individual patient. For example, in the first patient scenario, identifying the lack of benefit of chemotherapy given a low recurrencerisk score, eliminated the use of chemotherapy, decreased the net costs of care, and improved the patient's quality of life. The Genomics and Personalized Medicine Act of 2007 (S.976, available at http://www. thomas.gov/cgi-bin/query/z?c110:S.976. IS:), introduced by then-Senator Barack
Obama, has the stated purpose "to secure the promise of personalized medicine for all Americans by expanding and accelerating genomics research and initiatives to improve the accuracy of disease diagnosis, increase the safety of drugs, and identify novel treatments." This bill, which may fare better under a new administration, reflects an emerging national dialogue that is beginning to address both access to and development of advances in medical technologies.

With political and scientific energy supporting the development and application of such advances, are we to rush headlong toward expanded coverage of molecular and genomic technologies? How will we pay the costs of development and deployment of what are expensive tests? One could argue that the costs of caring for an individual patient may ultimately be lower when molecular and genomic technologies are incorporated into treatment decisions. From a societal standpoint, however, costs of medical care are likely to increase with expanded use of such technologies given the costs associated with such technologies. Providing specialized information and decision support further increases the expenses associated with these tests.

Moreover, molecular and genomic technologies are often adopted without rigorous evaluation ${ }^{3}$ or are used in settings or populations other than the ones for which they were intended. For example, despite conflicting data about the role of CYP2D6 polymorphisms and the lack of prospective data, insurance will generally cover the cost of CYP2D6 genotyping. Expanding insurance coverage of expensive tests will undoubtedly increase the demand for such tests among providers and patients even in cases of minimal or no benefit. Furthermore, direct-to-consumer marketing of genomic technologies will further increase demand for testing, as it has in the case of magnetic resonance imaging for breast cancer screening. Enthusiasm for new tests and technologies will, predictably, extend their use to people who fall outside the setting in which they were developed, increasing costs of care without corresponding improvements in health outcomes. Just because there are people (and insurers) able and willing to pay for a test does not necessarily mean that they should.

Evaluating the impact of these tests across a broad range of outcomes-not only survival and cost-effectiveness but also patient satisfaction, quality of life, knowledge, decision regret, and other decision-making outcomes-is warranted before we can know whether we can or should offer population-based testing approaches. It has been argued that improving the fidelity of established medical technologies-applying the right technology to the right people in the right way-will have a greater impact on the health of a population than will new technologies. ${ }^{4}$ That is, ensuring even distribution of "low-tech" biomarkers (for example, high-quality assessment of estrogen receptor status in primary breast cancers) may need to be our national priority over expanding access to newly discovered markers that have not yet found their proper place in prevention, diagnostic, and therapeutic algorithms. The collective behavior of our health-care system does not suggest any inclination to limit the use of expensive technologies. ${ }^{5}$ Our reluctance (and perhaps inability) to withhold any technology from our patients who are able to pay makes it even more important that we scrutinize the use of new and old technologies-whether predictive, diagnostic, prognostic, or therapeutic-along the entire continuum of prevention and medical care. As with new pharmaceutical agents, specific guidelines for the use of genomic technologies will increase their appropriate use; insurers can enforce compliance with guidelines by refusing to pay for technologies in patients or situations in which they have not proven to be effective. Strategic and comprehensive policies that address the optimal uptake of established tests while incorporating and identifying the correct use of emerging tests will increase the likelihood of credible, evidence-based delivery of care. Only in doing so will we reduce waste, reduce inequities, and achieve high-quality care for all.

\section{ACKNOWLEDGMENTS}

This work was funded in part by National Institutes of Health/National Cancer Institute grant R01 CA119202. 


\section{CONFLICT OF INTEREST}

The author declared no conflict of interest.

() 2009 ASCPT

1. Peterson-lyer, K. Pharmacogenomics, ethics, and public policy. Kennedy Inst. Ethics J. 18, 35-56 (2008).

2. Ancker, J.S. \& Kaufman, D. Rethinking health numeracy: a multidisciplinary literature review. J. Am. Med. Inform. Assoc. 14, 713-721 (2007).

3. Pepe, M.S., Feng, Z., Janes, H., Bossuyt, P.M. \&
Potter, J.D. Pivotal evaluation of the accuracy of a biomarker used for classification or prediction: standards for study design. J. Natl. Cancer Inst. 100, 1432-1438 (2008).

4. Woolf, S.H. \& Johnson, R.E. The break-even point: when medical advances are less important than improving the fidelity with which they are delivered. Ann. Fam. Med. 3, 545-552 (2005).

5. Mariner, W.K. Rationing health care and the need for credible scarcity: why Americans can't say no. Am. J. Public Health 85, 1439-1445 (1995).

\section{Educating for}

\section{Personalized Medicine:}

\section{A Perspective From Oncology}

\author{
DR Parkinson ${ }^{1}$ and J Ziegler ${ }^{1}$
}

\section{The personalized-medicine concept represents the future of oncology medicine. New genomics technologies will characterize patients biologically in ways that will drive more efficient and effective cancer treatment. Yet the introduction of these technologies is disruptive to current practices in clinical oncology, as well as to current regulatory and reimbursement strategies. The efficient introduction of personalized medicine will require education in addition to behavioral and policy changes by the various involved stakeholders.}

\section{Personalized medicine: premise and promise}

The concept of an individualized approach to patient treatment is not new in medicine. Treatment decisions for individual patients have always involved an integration of complex clinicopathological, technical, and socioeconomic considerations, and nowhere is this more important than in treatment decision making for cancer. When the evidence is supporting, oncologists have long been willing to base treatment decisions on laboratory measurements of a patient's tumor, the use of hormonal therapy in women with breast carcinoma being a decades-old example. The more recent premise of truly "personalized medicine," however, extends much further. The new genomics technology increasingly allows the characterization in detail of the relevant biology of an individual tumor and the patient with that tumor-potentially before, during, and after treatment. The clinical meaning of this biological characterization will come most efficiently from the careful study of patients while they are being treated with targeted therapeutics in clinical trials. Tumor classifications will be radically revised to reflect clinically relevant biology; they will be linked more closely with biologically targeted therapeutics use and will therefore inform treatment decisions more effectively. It is entirely possible that the concept of tumor "classification"-at least for the purposes of treatment decision making-will
Nodality, South San Francisco, California, USA. Correspondence: DR Parkinson (david.parkinson@nodality. com) evolve into one of tumor "characterization" at the individual patient level. ${ }^{1}$

This new concept of personalized oncology medicine affects every stakeholder in the cancer treatment community. How quickly the new biological information is transformed into better patient care and more efficient healthcare delivery will greatly depend on the education of these stakeholders regarding the meaning and the implications of personalized medicine.

\section{Educating for personalized medicine and overcoming the hurdles to its introduction}

The challenges regarding the development and introduction of the new genomic technologies and their potential for improvement of health care have been the focus of a number of recent activities and reports. The US Department of Health and Human Services (DHHS) Secretary's Advisory Committee on Genetics, Health, and Society (SACGHS) produced a report in May 2008 entitled "Realizing the Potential of Pharmacogenomics: Opportunities and Challenges" (http:// oba.od.nih.gov/oba/SACGHS/reports/ SACGHS_PGx_report.pdf).This report recognized the many challenges in integrating pharmacogenomic information into the health-care and public health systems and made a series of recommendations related in particular to the education and guidance of the multiple involved parties. Additionally, the President's Council of Advisors on Science and Technology (PCAST) produced a report, "Priorities for Personalized Medicine," in September 2008 (http://www.ostp.gov/ galleries/PCAST/pcast_report_v2.pdf). PCAST gathers advice from the private sector, including the academic community and industry. In its study, PCAST examined eight major policy areas affecting personalized medicine. Recommendations were made only in the areas of technology/tools, regulation, and reimbursement. Particularly with respect to the issues of physician and patient education and economics, the report pointed out that policy recommendations have not been made because personalizedmedicine product development is still in early stages. 